\title{
EFEITOS DO ESTABELECIMENTO \\ DE METAS PARA A AQUISIÇÃO \\ DE UMA HABILIDADE MOTORA
}

\begin{abstract}
GRAD. PRISCILA SILVA DE LIMA
Graduada em Educação Física pela Escola de Educação Física, Terapia Ocupacional e Fisioterapia da Uversidade Federal de Minas Gerais (EEFFTO/UFMG). Membro do Grupo de Estudos em Desenvolvimento e Aprendizagem Motora - GEDAM (Belo Horizonte - Minas Gerais - Brasil)

E-mail: pri.lima08@gmail.com
\end{abstract}

\section{DR. RODOLFO NOVELLINO BENDA}

Doutor em Educação Física pela Universidade de São Paulo. Professor Associado da Universidade Federal de Minas Gerais, sub-coordenador do Grupo de Estudos em Desenvolvimento e Aprendizagem Motora - GEDAM (Belo Horizonte - Minas Gerais - Brasil)

E-mail: rodolfobenda@yahoo.com.br

\section{MS. FABIANO DE SOUZA FONSECA}

Mestrado em Ciências do Esporte na Universidade Federal de Minas Gerais.

Membro do Grupo de Estudos em Desenvolvimento e Aprendizagem Motora - GEDAM

(Belo Horizonte - Minas Gerais - Brasil)

E-mail: fabianoef@gmail.com

\section{MS. FABRICIA QUINTÃO LOSCHIAVO-ALVARES}

Mestrado em Ciências do Esporte na Universidade Federal de Minas Gerais. Membro do Grupo de

Estudos em Desenvolvimento e Aprendizagem Motora - GEDAM

(Belo Horizonte - Minas Gerais - Brasil)

E-mail: fabriciato@yahoo.com.br

\section{MS. ALESSANDRA AGUILAR COCA UGRINOWITSCH}

Mestrado em Ciências do Esporte na Universidade Federal de Minas Gerais. Membro do Grupo de

Estudos em Desenvolvimento e Aprendizagem Motora - GEDAM

(Belo Horizonte - Minas Gerais - Brasil)

E-mail: cocaugri@hotmail.com

\section{GRAD. ALINE HORTA MIGUEL JUNQUEIRA}

Mestranda em Ciências do Esporte na Universidade Federal de Minas Gerais.

Membro do Grupo de Estudos em Desenvolvimento e Aprendizagem Motora - GEDAM

(Belo Horizonte - Minas Gerais - Brasil)

E-mail: alinehort@hotmail.com

\section{MS. MARIA FLÁVIA SOARES PINTO CARVALHO}

Mestrado em Ciências do Esporte na Universidade Federal de Minas Gerais.

Membro do Grupo de Estudos em Desenvolvimento e Aprendizagem Motora - GEDAM

(Belo-Horizonte - Minas Gerais - Brasil)

E-mail: totscarvalho@yahoo.com.br 


\section{DR. HERBERT UGRINOWITSCH}

Pós-doutorado na Universidade de Queensland, Austrália. Professor Adjunto da Universidade Federal de Minas Gerais. Co-coordenador do Grupo de Estudos em Desenvolvimento e Aprendizagem Motora - GEDAM

(Belo Horizonte - Minas Gerais - Brasil)

E-mail: herbertu@ufmg.br

\section{RESUMO}

O presente estudo investigou os efeitos do estabelecimento de metas de curto e de longo prazo na aprendizagem do arremesso de dardo de salão. O experimento foi conduzido em três etapas: pré-teste ( 10 tentativas); aquisição (60 tentativas), com manipulação da temporalidade da meta; e pós-teste (10 tentativas). Os 33 universitários destros foram divididos em três grupos: meta específica de curto prazo (GEC), meta específica de longo prazo (GEL) e o não meta (GNM). Na análise da precisão e da consistência, a ANOVA two way identificou diferença entre pré-teste e pós-teste e entre os blocos da aquisição. Apesar de todos os grupos terem aprendido a tarefa, não houve diferença entre eles. Sugere-se a realização de novos estudos sobre o tema.

PALAVRA-CHAVE: Aprendizagem motora; estabelecimento de metas; temporalidade da meta; arremesso de dardo de salão.

\section{INTRODUÇÃO}

De modo geral, é possível afirmar que as pessoas ficam mais empenhadas para sustentar suas ações e completar suas tarefas quando são motivadas. A estratégia de estabelecer metas é considerada uma importante estratégia de motivação para atingir um objetivo planejado e, dessa forma, organizar os esforços necessários para alcançá-lo. (BURTON, 1994). Por definição, meta é o que o indivíduo está tentando realizar; é o alvo ou o objetivo de uma ação. (UGRINOWITSCH; COCA UGRINOWITSCH, 2003). Do ponto de vista prático, uma meta focaliza padrões de excelência, como aumentar a renda mensal em $5 \%$, perder 10 quilos ou diminuir em 4 segundos o tempo de corrida de 100 metros. (WEINBERG, 1992). Esse comportamento é considerado um traço característico do ser humano, observado na capacidade de projetar virtualmente condições futuras para guiar ações presentes. (MARINHO et al., 2009).

A partir dos estudos realizados na Psicologia Organizacional, particularmente da indústria, e admitindo a similaridade entre as tarefas industriais e esportivas, visto que ambas envolvem ações mentais e físicas direcionadas a uma meta, por analogia, Locke e Latham ( 1985) sugeriram que resultados semelhantes poderiam ser observados no contexto da Educação Física e Esportes. Essa proposição deu um impulso inicial para pesquisas nessa área. 
Alguns aspectos, denominados "atributos" por Burton (1994), podem influenciar a efetividade dessa estratégia motivacional, sendo que dentre os mais investigados pode-se destacar a coletividade, o nível de dificuldade, a especificidade e a temporalidade da meta. Uma meta pode ser considerada coletiva ou de grupo quando estipulada para todo o grupo como uma unidade, e não a simples soma de metas individuais. (WEINBERG; WEIGAND, 1993). Apesar de se acreditar que o estabelecimento de metas coletivas deveria melhorar o desempenho de uma equipe (LOCKE; LATHAM, 1985), poucos estudos foram conduzidos para investigar a coletividade da meta e o desempenho de equipes esportivas. (DUTRA, 2007; UGRINOWITSCH; DANTAS, 2002). Ainda assim, os resultados da manipulação desse atributo indicam haver efetividade da meta de grupo no desempenho.

O grau de dificuldade da meta refere-se à diferença entre o nível de desempenho atual e o nível de desempenho estabelecido pela meta. (LOCKE; LATHAM, 2002). Esses autores, em seu trabalho de 1985, sugerem que metas difíceis, entretanto atingíveis, apresentariam melhor desempenho do que o de emprego de metas fáceis. Apesar de haver resultados de estudos de laboratório que dão suporte a essa hipótese (DUTRA; UGRINOWITSCH, 2005), no meio esportivo esses resultados são divergentes. (KYLLO; LANDERS, 1995).

Quanto à especificidade, a meta pode ser classificada em dois tipos: meta genérica (ex. faça o melhor possível) ou meta específica (ex: acerte 8 saques em I 0 tentativas). Locke e Latham ( 1985 ) apontam que metas específicas acarretariam em desempenhos superiores quando comparadas com metas genéricas. $\bigcirc$ efeito da especificidade da meta tem sido alvo de vários estudos, entretanto, Weinberg ( 1 992) ressalta que, no campo esportivo, os resultados não têm sido conclusivos.

Por fim, as metas também podem ser manipuladas temporalmente, isto é, as metas podem ser de longo prazo e de curto prazo. Weinberg (200l) propõe que metas de curto prazo são importantes aliadas às metas de longo prazo porque podem fornecer feedback sobre progressos relativos à meta de longo prazo. Esse feedback pode possuir uma função motivacional, além de permitir o ajuste da meta, dependendo da situação. Metas de curto prazo permitem que os participantes focalizem na melhora de incrementos menores, o que pode fazer as metas parecerem mais atingíveis do que metas de longo prazo, aparentemente impossíveis. Embora as metas de curto prazo sejam obviamente importantes, metas de longo prazo são também necessárias, pois auxiliam a manutenção da direção e do foco no objetivo final (WEINBERG, 1992). Locke e Latham (1990) apontam que o uso da meta de longo prazo combinada a metas de curto prazo levaria a um melhor desempenho, comparativamente ao uso de uma delas isoladamente. A meta muito longa parece perder o efeito motivacional e, nesse sentido, a união com metas de curto prazo 
auxiliaria na manutenção do foco na meta, mas a combinação desses dois aspectos temporais não tem sido alvo de muitos estudos. (MARINHO et al., 2009).

Até a década de 80, o foco principal das pesquisas sobre o estabelecimento de metas era o desempenho esportivo, com a utilização de tarefas que envolviam força, resistência, potência, entre outros (HALL et al., 1987; WEINBERG, 200 I). Somente na década de 90 houve um interesse maior sobre o efeito do estabelecimento de metas na aquisição de habilidades motoras, e sua influência no comportamento motor. No entanto, sob essa óptica a quantidade de estudos ainda é reduzida. (MARINHO et al., 2009).

Os aspectos temporais da meta têm recebido pouca atenção por parte dos pesquisadores da área de aprendizagem motora (MARINHO et al., 2009). Esse atributo foi investigado na tarefa de flutuação no meio líquido em sujeitos iniciantes (FREUDENHEIM; TANI, 1998), com grupo meta genérica, grupo meta específica de longo prazo e grupo meta específica combinada com metas de curto prazo. Os resultados não mostraram diferenças entre os grupos. Essa mesma questão foi investigada por Corrêa, Souza Junior, Perroti Junior (2002) na aprendizagem do passe no voleibol, mas com sujeitos experientes, divididos em quatro grupos: meta específica de longo prazo, meta específica de curto prazo, meta genérica e sem meta. A análise estatística também não apontou diferenças intergrupos.

No geral, os estudos relacionados à área de Aprendizagem Motora não identificaram efeito da temporalidade da meta, mas essa variável não levou a um pior desempenho. Sendo assim, o presente estudo pretende investigar os efeitos do estabelecimento de metas específicas de curto e longo prazo na aprendizagem de uma habilidade motora. Tendo em vista todo o referencial teórico, espera-se que a meta de curto prazo conduza a um melhor desempenho comparada a meta de longo prazo.

\section{MATERIAL E MÉTODOS}

Participaram desse estudo 33 sujeitos de ambos os sexos, voluntários, universitários, com média de idade 22,2 anos ( $\pm 3,2$ anos). Todos os voluntários eram inexperientes na tarefa e assinaram um termo de consentimento antes de iniciar o experimento. Todos os procedimentos da pesquisa foram de acordo com o Comitê de Ética da Universidade Federal de Minas Gerais.

Os instrumentos utilizados foram um alvo circular de sisal, com 10 circunferências circunscritas de $\mathrm{I} \mathrm{cm}$ de raio, cada uma delas pontuadas de $\mathrm{I}$ a 10 , sendo que o centro do alvo equivalia a 10 pontos e a circunferência mais externa, I ponto. Foram utilizados três alvos de salão (peso 20 gramas, comprimento $7 \mathrm{~cm}$ ). $\bigcirc$ alvo foi 
posicionado sobre uma esteira de borracha, com seu centro a uma distância de 2,5 m da área limite de lançamento, demarcada por uma fita no chão. Os lançamentos que não atingiram o alvo receberam pontuação 0 . No caso dos dardos caídos nas linhas divisórias das áreas de pontuação, foi atribuída a pontuação referente à área de maior valor. $\bigcirc$ alvo foi posicionado paralelamente ao solo, com o seu centro distante a 2,5 metros da área de lançamento dos dardos (FIGURA I). A tarefa consistiu em lançar o dardo com o movimento póstero-anterior do membro superior, abaixo da linha do quadril, com o objetivo de atingir o centro do alvo (FIGURA I). Esta tarefa foi adotada com base em estudos anteriores de aprendizagem motora que utilizaram a mesma tarefa, como o de Al-Abood et al. (200la) e também porque já foi utilizada em experimento de aprendizagem motora que manipulou esta mesma variável. Por último, esta tarefa é apropriada para testar a pergunta do estudo porque os sujeitos facilmente compreendem a tarefa e entendem facilmente a meta estabelecida. Essa tarefa foi adaptada de Al-Abood et al. (200 la), Al-Abood, Davids e Bennett (200 l b).

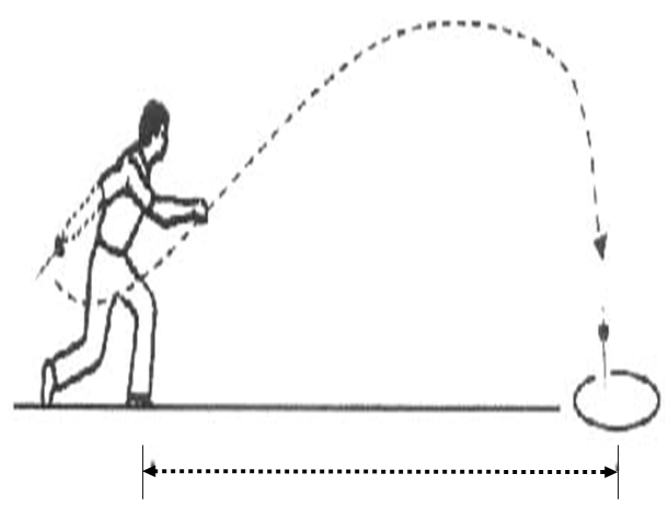

$2,5 \mathrm{~m}$

Figura I. Posicionamento do alvo na sala em relação ao indivíduo, tarefa adaptada de Al-Abood et al. (200 la), Al-Abood, Davis e Bennett, 200 l b).

\section{PROCEDIMENTOS}

O experimento foi conduzido em três etapas, em uma sala de coleta preparada para esse fim, na qual a coleta foi realizada individualmente. Inicialmente, foi realizado um pré-teste com 10 lançamentos de dardos e com a mesma meta de acertar o centro do alvo. $\bigcirc$ movimento a ser realizado foi demonstrado uma 
vez pelo experimentador. Posteriormente, teve início a fase de aquisição com 60 tentativas, na qual o aspecto temporal da meta foi manipulado. Dez minutos após o término da aquisição, os sujeitos realizaram 10 tentativas da mesma tarefa, porém sem informação em relação à meta. $\bigcirc$ número de tentativas das três etapas do presente experimento foi definido por estudo piloto.

Os 33 voluntários foram recrutados aleatoriamente, mas para garantir que os grupos do experimento partissem de níveis semelhantes de habilidade, eles foram divididos em três grupos homogêneos a partir do desempenho apresentado no pré-teste: grupo meta específica de curto prazo (GEC), grupo meta específica de longo prazo (GEL) e o grupo não meta (GNM). Para o GEC foram estipuladas 4 metas, de acordo com a média de pontuação das 10 tentativas do pré-teste, acrescentando 30\%, 40\%, 50\% e 60\%, respectivamente. O critério estipulado para mudança da meta foi alcançar ou superar a média da meta estabelecida em um bloco de 10 tentativas. $O$ GEL recebeu uma única meta específica, com acréscimo de $60 \%$ sobre a média de pontos obtida no pré-teste, a ser atingida até o final do experimento. Ao final de cada bloco de 10 tentativas, os grupos experimentais receberam informação sobre a pontuação média alcançada e em relação à meta. Para o GNM não foi estipulada nenhuma meta pelo experimentador.

Ao término da coleta, todos os sujeitos respondiam a um questionário com perguntas relacionadas ao autoestabelecimento de metas, e os grupos experimentais também eram questionados sobre o comprometimento e a avaliação subjetiva da dificuldade da meta estipulada. (MARINHO et al., 2009).

\section{TRATAMENTO DOS DADOS}

A análise dos efeitos da variável independente manipulada foi conduzida através das medidas de precisão e de dispersão do escore em blocos de dez tentativas, através do pacote estatístico Statistica for Windows 7.0. Os dados atenderam aos critérios de normalidade, por isso foi utilizada a ANOVA two way com medidas repetidas no segundo fator, uma na fase de aquisição (3 Grupos x 6 Blocos), e outra no pré-teste e no pós-teste (3 grupos $\times 2$ blocos). $O$ teste post hoc de Tukey foi utilizado para identificar possíveis diferenças, e o nível de significância adotado foi $P<0,05$.

\section{RESULTADOS}

Na análise da precisão dos arremessos, na comparação entre o pré-teste e o pós-teste (FIGURA 2), a ANOVA two way com medidas repetidas no último fator identificou diferença significativa no fator blocos $\left(F_{1,30}=12,762, p=0,001\right)$. O teste 
post hoc de Tukey mostrou que o pós-teste teve média de pontuação superior ao pré-teste. No entanto, não foram verificadas diferenças entre grupos ou interação entre blocos e grupos $(p<0,05)$. Na fase de aquisição, a ANOVA detectou diferença significativa no fator blocos $\left(F_{5,150}=2,989, p=0,013\right)$. $O$ teste post hoc de Tukey indicou que no bloco I, a precisão nos arremessos foi menor que nos blocos 3 $(p=0,0 \mid 9)$ e $4(p=0,048)$. Contudo, não foi detectado efeito significante entre os grupos ou interação entre os dois fatores $(p>0,05)$.

Para a medida de consistência (coeficiente de variabilidade), na comparação entre o pré-teste e o pós-teste, a ANOVA two way identificou diferença significativa entre blocos $\left(F_{6,180}=7,452, p=0,00 \mathrm{I}\right)$. $O$ teste post hoc de Tukey mostrou que o pré-teste foi mais variável que o pós-teste. No entanto, não foram verificadas diferenças significantes entre grupos ou interação entre blocos e grupos ( $p>0,05)$. Na fase de aquisição, a ANOVA two way apontou diferenças significativas entre blocos $\left(F_{6,180}=7,452, p=0,00 \mathrm{I}\right)$. O teste post hoc de Tukey indicou que o bloco I teve o coeficiente de variabilidade maior que os blocos 3, 4, 5 e 6, sendo que o último bloco da aquisição foi o mais consistente $(p<0,05)$. Contudo, não foram detectadas diferenças entre os grupos ou interação entre blocos e grupos $(p>0,05)$.

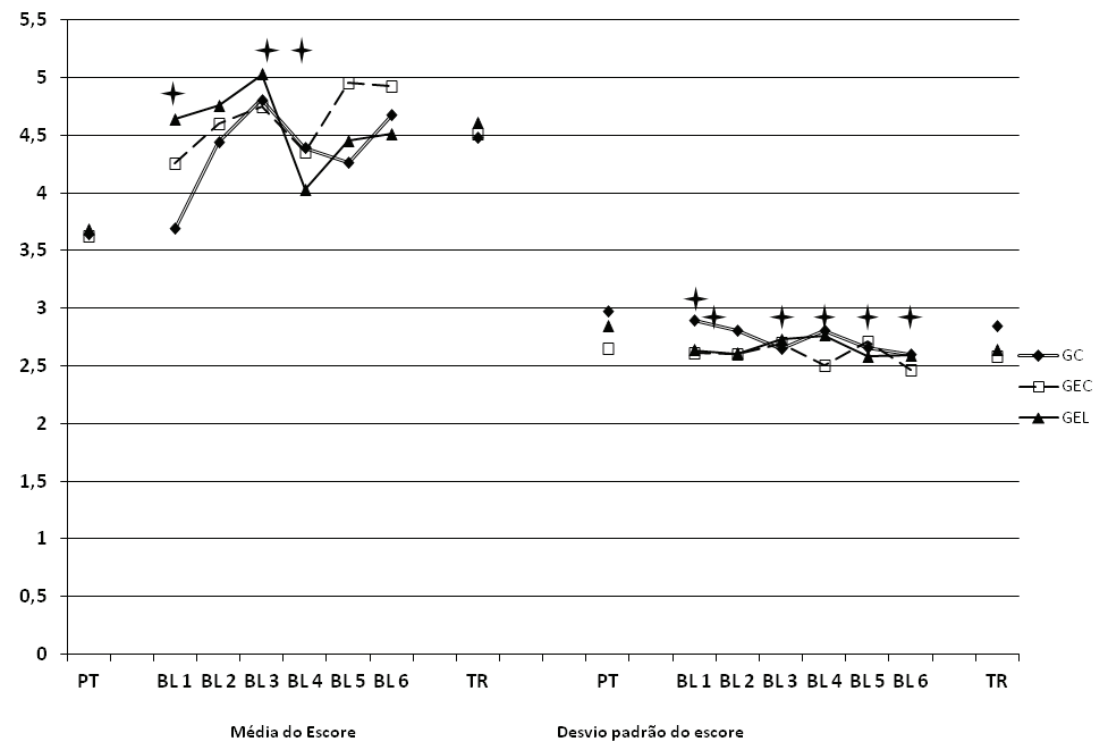

Figura 2. Média e desvio-padrão do escore em blocos de 10 tentativas no pré-teste (PT), fase de aquisição e teste de retenção (TR). 


\section{DISCUSSÃO E CONCLUSÕES}

O objetivo desse estudo foi investigar os efeitos do estabelecimento de metas de curto e longo prazo (temporalidade da meta) no processo de aprendizagem de uma habilidade motora discreta. Os indivíduos foram divididos em três grupos: grupo com meta de curo prazo (GEC), grupo com meta de longo prazo (GEL) e grupo não meta (GNM). Os resultados da análise da precisão dos arremessos e do coeficiente de variabilidade, apesar de mostrarem que os grupos aprenderam a tarefa praticada, não dão suporte às hipóteses de Locke e Latham (1985), de que o estabelecimento de metas, em especial de metas de curto prazo, acarretaria desempenho superior na aprendizagem.

O pré-teste foi comparado com o pós-teste para verificar diferenças na precisão e na consistência. Os resultados mostraram melhoras nesses dois parâmetros para os três grupos, o que indica que todos aprenderam a tarefa, independente da variável manipulada. Durante a fase de aquisição, a progressão do desempenho ocorreu de forma semelhante em todos os grupos, não havendo diferenças entre eles. Já a variabilidade reduziu durante essa fase, sendo que a partir do terceiro bloco os grupos ficaram mais estáveis.

Uma possível explicação para a ausência de diferenças entre os grupos pode ter sido a própria característica da tarefa, que permitia aos sujeitos visualizar a pontuação no alvo, obtendo informação a cada lançamento. Esse feedback pode ter favorecido o auto-estabelecimento de metas por parte do grupo sem meta, assim como dos demais grupos. (WEINBERG et al., 1988). Conforme foram atingindo a meta, é possível que os sujeitos logo tenham autoestabelecido outras, inclusive aqueles com meta de longo prazo. Em alguns casos, a meta estipulada foi rapidamente alcançada, o que pode ter levado os sujeitos a estabelecer novas metas mais desafiadoras. Por outro lado, para outros indivíduos a meta estipulada pode ter sido considerada muito distante e difícil, favorecendo o autoestabelecimento de metas mais atingíveis. Sugere-se que futuros estudos controlem o autoestabelecimento de metas no arremesso de dardos por meio de questionários que identifiquem o comprometimento dos sujeitos em relação à meta estabelecida pelo experimentador.

O grau de comprometimento com a meta estabelecida também é um fator importante a ser considerado. Segundo Locke e Latham (1985), as metas devem ser aceitas pelos sujeitos para que influenciem de modo positivo no desempenho. Possivelmente a meta estabelecida não tenha sido efetivamente incorporada pelos sujeitos. Assim, a meta fornecida pode não ter coincidido com a meta perseguida pelos sujeitos, anulando então a diferença das condições experimentais. 
Alguns estudos apontam para a existência de alguma relação entre a complexidade da tarefa e o efeito da meta sobre o desempenho. (BURTON, 1994; UGRINOWITSCH; DANTAS, 2002). Freudenheim e Tani ( 1998) não verificaram o efeito do estabelecimento de metas em uma tarefa de flutuação no meio líquido, o que pode ter ocorrido devido ao nível de complexidade da tarefa. Neste estudo, a tarefa de lançamento de dardo de salão não apresenta um nível de complexidade muito alto quando comparado com habilidades esportivas, não permitindo que aparecesse o efeito da variável independente manipulada. Porém, mais estudos precisam ser realizados para chegar-se a conclusões em relação à complexidade da tarefa.

Além dos fatores citados acima, outro aspecto a ser considerado é o nível de habilidade do sujeito. Ugrinowitsch e Coca Ugrinowitsch (2003) sugerem que metas relacionadas ao produto devem ser utilizadas quando os sujeitos já possuem experiência na habilidade. Na fase inicial de aprendizagem, sugere-se a utilização de metas relacionadas ao processo de execução da habilidade. Outra possibilidade para estudos futuros seria investigar essa mesma questão (temporalidade da meta) com sujeitos experientes.

Sugere-se testar esse pressuposto com tarefas que permitam controlar mais o acesso ao feedback durante o processo de aprendizagem, buscando inibir o estabelecimento de metas próprias. Aspectos como o nível de complexidade da tarefa e o nível de experiência dos indivíduos também merecem mais investigação.

Effects of goal setting for the acquisition of motor skills.

ABSTRACT: The present study investigated the effects of short and long-term goal setting in the learning of saloon dart throwing. The experiment was conducted in three stages: pretest ( 10 trials), acquisition ( 60 trials), with manipulation of the temporality of the goal, and post-test ( 10 trials). The 33 right-handed university students were divided into three groups: specific short-term goal (GEC), specific long-term goal (GEL) and no-goal (GNM). In the analysis of accuracy and consistency the two way ANOVA identified significant effect between pretest and post-test and between acquisition blocks as well. Although all the groups have learned the task, there was no difference between them. It is suggested further researches on the subject.

KEYWORDS: Motor Learning; goal setting; temporality goal settings; saloon throwing darts.

Efectos de la fijación de metas para la adquisición de habilidades motoras RESUMEN: El presente estudio investigó los efectos de la meta a corto y largo plazo de puesta enel aprendizaje del salón de lanzar dardos. El experimento se llevó a cabo en tres etapas: pre-test (10 ensayos), la adquisición (60 ensayos), con la manipulación de la 
temporalidad de la meta, y después de la prueba (10 ensayos). Los 33 estudiantes universitarios de la mano derecha se dividieron en tres grupos: objetivo concreto a corto plazo (GEC), el objetivo específico a largo plazo (GEL) y no se meta (GNM). En el análisis de la exactitud y la consistencia de dos vías ANOVA identificó un efecto significativo entre la pre y post-test y entre bloques de adquisición así. A pesar de todos los grupos han aprendido la tarea, no hubo diferencias entre ellos.

PALABRAS CLAVE: Aprendizaje motor; motor de la adquisición de habilidades; fijación de la meta; Salón Lanzar Dardos

\section{REFERÊNCIAS}

$\mathrm{AL}-\mathrm{ABOOD}, \mathrm{S}$. A. et al. Specifity of task constraints and effects of visual demonstrations and verbal instructions in directing learners' search during skill acquisition. Journal of Motor Behavior, Washington, v. 33, n. 3, p. 295-305, 200 la.

AL- ABOOD, S. A.; DAVIDS, K.; BENNETT, S. J. Effects of manipulating relative and absolute motion information during observational learning of an aiming task. Journal of Sports Science, Washington, v. 19, n. 7, p. 507-520, $200 \mathrm{lb}$.

BURTON, D. Goal setting in sport. In: SINGER, R. N.; TENNANT, L. R. (Ed.). Handbook of research of sport psychology. New York: MacNillan, 1994. p. 467-49I.

CORREAA, U. C.; SOUZA JUNIOR, O. P.; PERROTI JUNIOR, A. Os efeitos do estabelecimento de metas na aquisição de habilidades motoras em indivíduos em estágios avançados de aprendizagem. In: CONGRESSO BRASILEIRO DE COMPORTAMENTO MOTOR, 2., 2002, Gramado. Anais... Gramado, 2002. p. 54.

DUTRA, L. N. Efeito da dificuldade da meta de grupo na aprendizagem de habilidades motoras. 2007. Dissertação (Mestrado em Ciências do Esporte) - Escola de Educação Física, Fisioterapia e Terapia Ocupacional, Universidade Federal de Minas Gerais, Belo Horizonte 2007.

DUTRA, L. N..; UGRINOWITSCH, H. Efeito do Estabelecimento de diferentes percentuais de meta na aprendizagem de uma tarefa de posicionamento. The FIEP Bulletin, Foz do Iguaçu, v. 75, n. I, p. 154-157, 2005.

FREUDENHEIM, A.; TANI, G. O efeito do estabelecimento de metas na aquisição de uma habilidade de sustentação no meio líquido em crianças. Revista da APEF, Londrina, v. I3, n. I, p. 5-। I, 1998.

HALL, H.; WEINBERG, R.; JACKSON, A. Effects of goal specificity, goal difficulty and information feedback on endurance performance. Journal of Sports Science, Oxford, v. 9, n. 3, p. 43-54, 1987.

KYLLO, L. B.; LANDERS, D. M. Goal setting in sport and exercise: a research synthesis to resolve the controversy. Journal of Sport and Exercise Psychology, Champaign, v. 17, n. 2, p. | 17-137, 1995.

LOCKE, E. A. Problems with goal-setting research in sports: and their solution. Journal of Sport and Exercise Psychology, Champaign, v. 13, n. 3, p. 31।-316, 1991. 
LOCKE, E. A.; LATHAM, G. F. P. The application of goal setting to sports. Journal of Sport Psychology, Oxford, v. 7, n. 3, p. 205-222, 1985.

A theory of goal setting and task motivation. Englewood Cliffs: Prentice Hall, 1990.

Building a practically useful theory of goal setting and task motivation, a 35-year odyssey. American Psychological, Washington, v. 57, n. 9, p. 705-7I7, 2002.

MARINHO, N. F. S. et al. Estabelecimento de metas impostas pelo experimentador e autoestabelecidas: efeitos na aprendizagem do arremesso de dardo de salão. Revista de Educação Física, Maringá, v. 20, n. 4, p. 509-517, 2009.

UGRINOWITSCH, H.; DANTAS, L. E. T. B. P. Efeito do estabelecimento de metas na aprendizagem do arremesso do basquetebol. Revista Portuguesa de Ciência do Desporto, Porto, v. 2, n. 5, p. 58-63, 2002.

UGRINOWITSCH, H.; COCA UGRINOWITSCH, A. A. O efeito do estabelecimento de metas no treinamento esportivo. In: GARCIA, E. S.; LEMOS, K. L. M. Temas Atuais VIII em Educação Física e Esportes. Belo Horizonte: Health, 2003. p. 6I-72.

WEINBERG, R. S. Goal setting and motor performance: a review and critique. In: ROBERTS, G. C. (Ed.). Motivation in sport and exercise. Champaign: Human Kinetics, 1992. p. I77- 197.

Motivation in sport and exercise: the special case of goal-setting. IN: WORLD CONGRESS OF SPORTS PSICHOLOGY, I0. Annals... Skiathos, Hellas, 2001.

WEINBERG, R. S.; BRUYA, L.; LONGINO, J.; JACKSON, A. Effect of goal proximity and specificity on endurance performance of primary-grade children. Journal of Sport and Exercise Psychology, Champaign, v. 10, n. I, p. 81-91, 1988.

WEINBERG, R. S.; WEIGAND, D. A. Goal setting and exercise: a reaction to Locke. Journal of Sport and Exercise Psychology, Champaign, v. I 5, n. I, p. 88-96, 1993.

WIDMEYER, W. N.; DUCHARME, K. Team building through team goal setting. Journal of Applied Sport Psychology, Champaign, v. 9, n. I, p. 97-1 13, 1997.

Recebido:09 jun. 201 I

Aprovado: 23 mar. 2012

Endereço para correspondência:

Herbert Ugrinowitsch

Av. Antônio Carlos, 6627

Universidade Federal de Minas Gerais, EEFFTO

Bairro Pampulha

Belo Horizonte - MG

CEP: 3 |270-90 | 